\title{
Atypical Hemolytic Uremic Syndrome
}

National Cancer Institute

\section{Source}

National Cancer Institute. Atypical Hemolytic Uremic Syndrome. NCI Thesaurus. Code C123223.

Hemolytic uremic syndrome not associated with shiga toxin-producing enterobacteria. 